\title{
Fairness Index Based on Variational Distance
}

\author{
Jing Deng ${ }^{\dagger} \quad$ Yunghsiang S. Han ${ }^{\ddagger \S}$ and Ben Liang* \\ ${ }^{\dagger}$ Dept. of Computer Science, University of North Carolina at Greensboro, Greensboro, NC, USA. jing.deng@uncg.edu \\ ${ }^{\ddagger}$ Graduate Inst. of Communication Engineering, National Taipei University, Taiwan. yshan@ mail.ntpu.edu.tw \\ $\S$ Dept. of Computer Science and Information Engineering, National Chi Nan University, Taiwan. \\ *Dept. of Electrical and Computer Engineering, University of Toronto, Toronto, Canada. liang@comm.utoronto.ca
}

\begin{abstract}
Fairness index among competing hosts in communication networks is an important system measurement. Several fairness index measurements have been proposed in the technical literature. However, most of these measurements, such as the max/min fairness index and Jain's index, reflect only a long-term average fairness of the system. Instantaneous fairness property has not been captured. In this paper, we propose a new fairness index to reflect such short-term fairness and long-term fairness at the same time. Comparisons of our proposed fairness index, termed Fairness Index based on Variational Distance (FIVD), and related fairness indices are presented to show the benefit of our measurement.
\end{abstract}

\section{INTRODUCTION}

In communication networks and computer systems, multiple agents often share common resources and services. The fairness in such resource and service sharing is an important system performance metric. For example, in wireless communication networks, several hosts often share a single wireless channel following a Medium Access Control (MAC) protocol. Without considering fairness among different hosts, a MAC protocol designed to maximize the system throughput might assign the channel to just one busy host for a long period of time. Such domination of the channel by a single host would be unfair to all other competing hosts, which would experience longer access delays and a reduced share of the channel throughput. This example illustrates a general tradeoff between system efficiency and fairness. In the case of MAC, a balanced design should allow fair competition among the hosts, even though doing so might reduce the overall throughput. To allow judicious system design based on the tradeoff between efficiency and fairness, one must properly measure these two quantities.

Unlike the obvious metrics for system efficiency, fairness is harder to define in general. It may depend on specific system modeling assumptions and application requirements. For example, the well-known General Processor Sharing (GPS) model dictates that each host is assigned a fair portion of the shared resource at any time interval [1]. In contrast, under some application scenarios, better throughput-fairness tradeoff could be achieved, by first guaranteeing a basic fairness level such that a minimum application throughput demand in each host is satisfied, and then increasing the resource share of some hosts to improve the overall system throughput [2]. In this work, we consider strict fairness in the sense of GPS. Furthermore, we aim for basic equal-share fairness instead of weighted fairness.
Given a resource allocation schedule, a fairness index is a real number that measures how fair or unfair the resource is shared among the competing hosts. For example, Jain's fairness index [3] is given by

$$
F I=\frac{\left(\sum x_{i}\right)^{2}}{N \cdot \sum x_{i}^{2}},
$$

where $x_{i}$ is the access share of host $i$ among $N$ competing hosts. Another well-known example is the max-min index [4][5], which gives the ratio between the maximum access share by a host and the minimum access share by a host. Both Jain's index and the max-min index are designed to measure fairness in the long run. A resource allocation schedule in a system with $N$ competing hosts is considered long-term fair if the proportion of each host accessing the resource is $1 / N$ over a long period of time.

A resource allocation schedule in a system with $N$ competing hosts is considered short-term fair if the relative probability of each host accessing the resource is $1 / N$ in any short interval. It is clear that short-term fairness implies longterm fairness. In contrast, even if an allocation schedule is long-term fair, it may not be short-term fair. For example, a MAC scheduler that admits host transmissions in bursts may be able to maintain fair medium sharing in the long run, but in the short term, some hosts experience longer delay than the others. Currently there is no widely adopted metric for shortterm fairness in the literature. Existing studies on resource scheduling algorithms use a variety of indices. They adopt either a sliding-window version of Jain's index [6], an interaccess counting approach [7], or other metrics defined in an ad hoc manner without in-depth justification [8].

In this work, we promote a more systematic understanding of the metric for fairness. We propose a new index, termed Fairness Index based on Variational Distance (FIVD), which captures the average fairness as a sequence of resource allocation that grows in length. By doing so, it allows the combination of cascading fairness metrics at different time durations. We show that FIVD has the intrinsic properties of symbol-level granularity and perfect representation. Furthermore, it is bounded in value, independent of the population size, and independent of the unit of measurement. We further compare FIVD with Jain's index and the max-min index using sample sequences to demonstrate the relative advantages of FIVD. Finally, we conduct ns-2 simulation of the IEEE 802.11 DCF MAC scheme to illustrate how FIVD can provide design 
guidelines for fair resource allocation in practical systems.

The rest of this paper is organized as follows. Section II summarizes the related work. In Section III, we define FIVD and present its properties. Comparative and simulation studies are given in Section IV. Finally, we give concluding remarks in Section V.

\section{RELATED WORK}

The concept of fairness is prevalent in communication networks and computer systems. See for example a survey in [9], where it is suggested that a main problem in intranetworking is to eliminate unfairness inherent in the MAC mechanisms. The most direct fairness definitions such as GPS [1] and basic fairness [2] have binary outcomes. Under these definitions, a resource allocation schedule is either fair or not. In this work, we are interested in quantifying the fairness level when the schedule is not perfectly fair.

Toward this goal, various fairness measurements have been proposed that are derived from several different distance measures between the allocation schedule and an idealized schedule. Often the idealized schedule is network topology, application, and cost dependent. Examples of these include direct data flow throughput comparison [10][11], delay variation [12], normalized throughput and delay differentials [8], proportional fairness based on pricing [12][13], and utility based fairness [14]. In this work, our goal is to design a fairness metric based on a resource allocation sequence alone and independent of the system operation considerations.

One of the most often used long-term fairness metric is Jain's Index [3]. As shown in (1), it approximates the ratio between the squares of the first and second moments of access share $x_{i}$. Since $x_{i}$ is aggregated over time, Jain's index does not quantify short-term fairness. The max-min index is also commonly used in the literature [4][5]. It is clear that this index cannot be used in a very short time period, since the minimum access share approaches zero in this case.

From an information theoretic point of view, short-term fairness measures the randomness of the resource allocation schedule. Hence, the joint entropy [15] of the allocation sequence could be used as a fairness index. However, it would require multiple realizations of the allocation sequence to compute the joint entropy. Therefore, this metric could be used to quantify only the fairness of a scheduler, not a scheduling sequence.

A solution to adapt Jain's index for short-term fairness is to evaluate the average of Jain's index for sliding windows with a short duration [6]. However, there is no formal analysis on the suitability of this approach. In particular, as the window size becomes smaller, the access share estimation becomes increasingly inaccurate. An alternative is to count the number of resource accesses by other hosts between two consecutive accesses by a host. In [7], it is proposed that the distribution of such inter-access count should be compared with an ideal distribution. However, the comparison is quantified only by a few simple statistics, such as the mean value, drawn from those two distributions. In this work, we systematically study FIVD, demonstrating its intrinsic properties such as symbol-level granularity, perfect representation, boundedness, independence of the population size, and independence of the unit of measurement.

\section{FAIRnESS INDEX BASED ON VARIATIONAL Distance}

\section{A. Definition of FIVD}

Let $\boldsymbol{X}$ be the set of all hosts and $N=|\boldsymbol{X}|>1$ be the number of elements of $\boldsymbol{X}$. Let $T$ be a sequence of the elements of $\boldsymbol{X}$ with length $L$ and $T(b)$ be the sub-sequence of $T$ that contains the $b$ elements starting from position 1 in $T, b=$ $1,2, \ldots, L$.

Define a probability distribution $\boldsymbol{P}_{T(b)}(x)$ on $T(b)$ as follows:

$$
\boldsymbol{P}_{T(b)}(x)=\frac{\text { the number of occurrence of } x \text { in } T(b)}{b},
$$

where $x \in \boldsymbol{X}$. We call $\boldsymbol{P}_{T(b)}(x)$ the occurrence probability of $x$.

We define the Occurrence Fairness Index $(\mathrm{OFI})^{1}$ of $T(b)$ with the help of variational distance [15] between $\boldsymbol{P}_{T(b)}(x)$ and the uniform distribution,

$$
\boldsymbol{U}(x)=1 / N \text { for all } x \in \boldsymbol{X},
$$

which is considered the most fair sequence.

The OFI of sequence $T(b)$ is defined as

$$
\begin{aligned}
\mathrm{OFI}(T(b)) & =\sum_{x \in \boldsymbol{X}}\left|\boldsymbol{P}_{T(b)}(x)-\boldsymbol{U}(x)\right| \\
& =\sum_{x \in \boldsymbol{X}}\left|\boldsymbol{P}_{T(b)}(x)-\frac{1}{N}\right|,
\end{aligned}
$$

where $|\cdot|$ calculates the absolute values. It is easy to see that $\operatorname{OFI}(T(b))$ is always non-negative and is zero if and only if $\boldsymbol{P}_{T(b)}(x)$ is the uniform distribution.

We have the following observations upon OFI:

1) The OFI of a sequence $T$ is the same as that of any permutation of $T$. Therefore, OFI only captures the occurrence frequency of each element, but not their positions in the sequence.

2) The OFI of a sequence with length $b=k N$, where $k$ is a non-negative integer, reaches its minimum value 0 when each of the elements appears $k$ times.

A resource access sequence $T$ of length $L$ is generated sequentially in time, starting from one element to $L$ elements, where each element denotes the host that is allocated the resource. The sub-sequences, $T(1), T(2), \ldots, T(L-1), T(L)=$ $T$, are generated subsequently. This suggests that the fairness of the sequence $T$ should be taken as the average of the OFI values of the above subsequences. Hence, we define the

\footnotetext{
${ }^{1}$ A more precise term should be the Occurrence Unfairness Index, but we follow the definitions of other earlier works and use the term "fairness."
} 
fairness, termed Fairness Index based on Variational Distance (FIVD), of a sequence $T$ with length $L>1$ as $^{2}$

$$
\operatorname{FIVD}(T)=\frac{1}{L-1} \sum_{i=2}^{L} \frac{\mathrm{OFI}(T(i))-\psi(i, N)}{\Psi(N)-\psi(i, N)}
$$

where

$$
\begin{aligned}
\Psi(N)= & 2-2 / N, \\
\psi(i, N)= & 2 r\left[\frac{k+1}{i}-\frac{1}{N}\right\rfloor, \\
& \quad \text { with } k=\left\lfloor\frac{i}{N}\right\rfloor, r=i-k N .
\end{aligned}
$$

Note that $\psi(i, N)$ and $\Psi(N)$ are the smallest and largest values of $\mathrm{OFI}(T(i))$, respectively. The reasons for $\psi(i, N)$ and $\Psi(N)$ will become clear in our analysis in Section III-B.

\section{B. Analysis}

We show two lemmas before presenting four theorems on FIVD.

Lemma 1: Let $\boldsymbol{P}_{1}$ and $\boldsymbol{P}_{2}$ be two distributions over $\boldsymbol{X}$. Then

$$
\sum_{x \in \boldsymbol{X}}\left|\boldsymbol{P}_{1}(x)-\boldsymbol{P}_{2}(x)\right|=2 \sum_{x \in \boldsymbol{V}}\left(\boldsymbol{P}_{1}(x)-\boldsymbol{P}_{2}(x)\right),
$$

where $\boldsymbol{V}$ is the largest subset of $\boldsymbol{X}$ such that $\boldsymbol{P}_{1}(x)>\boldsymbol{P}_{2}(x)$ for each $x \in \boldsymbol{V}$.

Proof: See [15].

Corollary 1: OFI can be computed with those elements with occurrence probability higher than $1 / N$.

Proof: Applying $\boldsymbol{P}_{T}$ as $\boldsymbol{P}_{1}$ and $\boldsymbol{U}$ as $\boldsymbol{P}_{2}$ in Lemma 1, we can easily see that OFI of a sequence $T$ can be computed with those elements that appear more than $1 / N$.

Lemma 2: Let $T(b)$ be a given sequence on $\boldsymbol{X}$ and $T(b) x$ be the concatenation of two sequences $T(b)$ and $x$. If $\boldsymbol{P}_{T(b)}\left(x_{1}\right) \leq \boldsymbol{P}_{T(b)}\left(x_{2}\right)$, then $\operatorname{FIVD}\left(T(b) x_{1}\right) \leq$ $\operatorname{FIVD}\left(T(b) x_{2}\right)$.

Proof: Let $T_{1}=T(b) x_{1}$ and $T_{2}=T(b) x_{2}$. By definition,

$$
\begin{aligned}
& \operatorname{FIVD}\left(T_{1}\right)-\operatorname{FIVD}\left(T_{2}\right) \\
= & \frac{(b-1) \operatorname{FIVD}(T)}{b}+\frac{\operatorname{OFI}\left(T_{1}\right)-\psi(b+1, N)}{b(\Psi(N)-\psi(b+1, N))} \\
& -\frac{(b-1) \operatorname{FIVD}(T)}{b}-\frac{\operatorname{OFI}\left(T_{2}\right)-\psi(b+1, N)}{b(\Psi(N)-\psi(b+1, N))} \\
= & \frac{1}{b(\Psi(N)-\psi(b+1, N))}\left\{\operatorname{OFI}\left(T_{1}\right)-\operatorname{OFI}\left(T_{2}\right)\right\} .
\end{aligned} .
$$

\footnotetext{
${ }^{2}$ We leave out the case of $i=1$ since it provides no information for fairness. Also it can be proved that $\Psi(N)>\psi(i, N)$ for $2 \leq i \leq L$.
}

Since $\frac{1}{b(\Psi(N)-\psi(b+1, N))}>0$ we only need to consider $\operatorname{OFI}\left(T_{1}\right)-\operatorname{OFI}\left(T_{2}\right)$.

$$
\begin{aligned}
& \operatorname{OFI}\left(T_{1}\right)-\operatorname{OFI}\left(T_{2}\right) \\
= & \sum_{x \in \boldsymbol{X}}\left|\boldsymbol{P}_{T_{1}}(x)-\frac{1}{N}\right|-\sum_{x \in \boldsymbol{X}}\left|\boldsymbol{P}_{T_{2}}(x)-\frac{1}{N}\right| \\
= & \left|\frac{b \boldsymbol{P}_{T(b)}\left(x_{1}\right)+1}{b+1}-\frac{1}{N}\right|+\left|\frac{b \boldsymbol{P}_{T(b)}\left(x_{2}\right)}{b+1}-\frac{1}{N}\right| \\
& -\left|\frac{b \boldsymbol{P}_{T(b)}\left(x_{1}\right)}{b+1}-\frac{1}{N}\right|-\left|\frac{b \boldsymbol{P}_{T(b)}\left(x_{2}\right)+1}{b+1}-\frac{1}{N}\right| .
\end{aligned}
$$

There are four cases that need to be considered and we omit the proof due to page limitation.

The following Theorem underlines the intuitiveness of the FIVD definition in (3). It shows that, in order to reduce FIVD (or achieve better fairness), an element with the lowest occurrence probability should appear next. Therefore, assuming that each host has the same priority to use the shared resource (e.g., wireless channel), the host with the lowest occurrence probability should use the shared channel next in order to maintain fairness among all hosts.

Theorem 1: If $y$ is the element with the least occurrence probability in sequence $T(b)$, then $T(b+1)=T(b) y$ has the lowest FIVD among all possible one-element extension of $T(b)$ with an element in $\boldsymbol{X}$.

Proof: Directly from Lemma 2.

Theorem 2 helps to determine the patterns of sequence with the best fairness (i.e., smallest FIVD). Let $L=k N+r$, where $r=0,1, \ldots N-1$. Define $\Pi$ as the set of all permutations of $\boldsymbol{X}$, which has exactly $N$ elements.

Theorem 2: Let $A_{T}(L)$ be a set containing all sequences $T$ with length $L$ such that

$$
\begin{aligned}
T= & \left\{t_{1}, t_{2}, \ldots, t_{N}, t_{N+1}, \ldots, t_{2 N}, t_{2 N+1}, \ldots, t_{k N},\right. \\
& \left.t_{k N+1}, \ldots, t_{L}\right\}
\end{aligned}
$$

where $\left\{t_{\ell N+1}, \ldots, t_{(\ell+1) N}\right\} \in \Pi$ is a permutation of $\boldsymbol{X}$ for all $\ell \in\{0,1, \ldots, k-1\}$, and $\left\{t_{k N+1}, \ldots, t_{k N+r}\right\}$ is the first $r$ elements of any permutation of $\boldsymbol{X}$. Then, for any $T \in A_{T}(L)$,

$$
\operatorname{FIVD}(T)=0 \text {. }
$$

Proof: It is easy to see that $\operatorname{OFI}(T(\ell N))=0$ for all $\ell=1,2, \ldots, k$ because all elements in $\boldsymbol{X}$ appear in $T(\ell N)$ with exact $\ell$ times. Now consider $\operatorname{OFI}(T(\ell N+j))$ for $j=$ $1,2, \ldots N-1$. It is clear that $\boldsymbol{P}_{T(\ell N+j)}(x)=\frac{\ell}{\ell N+j}$ is less than $1 / N$ when $x$ does not appear in the last $j$ elements of $T(\ell N+j)$. By Lemma 1 ,

$$
\mathrm{OFI}(T(\ell N+j))=2 j\left[\frac{\ell+1}{\ell N+j}-\frac{1}{N}\right],
$$

which is equal to $\psi(\ell N+j, N)$. Hence, all terms in the summation of (3) are zero and $\operatorname{FIVD}(T)=0$.

Next we show that $T \in A_{T}(L)$ has the best FIVD among all sequences with length $L$, such that FIVD is never negative.

Theorem 3: A sequence $T$ of length $L$ has the best fairness among all sequences of length $L$ if and only if $T \in A_{T}(L)$. 
Proof: It is sufficient to prove that $\operatorname{OFI}(T(\ell N+j))$ is smallest among OFIs of all sequences with length $\ell N+j$, where $0<j<N$.

Let $T^{(0)}(\ell N+j)$ be any arbitrary sequence which is not in $A_{T}(\ell N+j)$. Recall that $\boldsymbol{P}_{T(\ell N+j)}(x)$ is $\ell /(\ell N+j)$ or $(\ell+$ $1) /(\ell N+j)$ for any $x \in \boldsymbol{X}$. Let $a$ and $b$ be elements with the largest and the smallest occurrence probabilities in $T^{(0)}(\ell N+$ $j)$, respectively. Clearly, $\boldsymbol{P}_{T^{(0)}(\ell N+j)}(a)>(\ell+1) /(\ell N+j)>$ $1 / N$ and $\boldsymbol{P}_{T^{(0)}(\ell N+j)}(b)<\ell /(\ell N+j)<1 / N$. Then we construct a new sequence $T^{(1)}(\ell N+j)$ by replacing every occurrence of $a$ with $b$ in $T^{(0)}(\ell N+j)$. Then $\boldsymbol{P}_{T^{(1)}(\ell N+j)}(a) \geq(\ell+$ $1) /(\ell N+j)>1 / N$ and $\boldsymbol{P}_{T^{(1)}(\ell N+j)}(b) \leq \ell /(\ell N+j)<1 / N$. By Lemma $1, \operatorname{OFI}\left(T^{(1)}(\ell N+j)\right)<\operatorname{OFI}\left(T^{(0)}(\ell N+j)\right)$ since $\boldsymbol{P}_{T^{(0)}(\ell N+j)}(b)$ still does not contribute to $\operatorname{OFI}\left(T^{(1)}(\ell N+j)\right)$ and $\boldsymbol{P}_{T^{(1)}(\ell N+j)}(a)$ is smaller. We can continue to lower $\operatorname{OFI}\left(T^{(n)}(\ell N+j)\right)$ to $\operatorname{OFI}\left(T^{(n+1)}(\ell N+j)\right)$ in the same fashion until a sequence $T^{(m)}(\ell N+j)$ whose largest occurrence probability of any element is equal to $(\ell+1) /(\ell N+j)$. In this case, $\operatorname{OFI}\left(T^{(m)}(\ell N+j)\right)=\operatorname{OFI}(T(\ell N+j))$. In combining with the fact that $\operatorname{OFI}(T(\ell N))=0$, the FIVD of $T$ is the smallest among all sequences with length $L$.

Theorem 4: The value of FIVD for any sequence $T$ of length of $L$ with $N$ competing hosts satisfies

$$
\operatorname{FIVD}(T) \leq 1
$$

Proof: We need to prove that the upper bound of FIVD is achieved when the sequence contains only one out of the $N$ hosts. First we prove that the OFI of any length is bounded above by $2-2 / N$. Let $T$ be an arbitrary sequence over $\boldsymbol{X}$ with length $L$. If $T$ contains $L$ of the same elements, by Lemma 1 , $\operatorname{OFI}(T)=2(1-1 / N)=2-2 / N$. Assume that there are more than one elements from $\boldsymbol{X}$ in $T$. Let $a$ and $b$ be elements with the largest and the smallest occurrence probabilities in $T$, respectively. Then $\boldsymbol{P}_{T}(a) \geq 1 / N$ and $0<\boldsymbol{P}_{T}(b) \leq 1 / N$. We then replace every occurrence of $b$ in $T$ with $a$ to obtain a new sequence $T^{\prime}$. According to Lemma $1, \operatorname{OFI}\left(T^{\prime}\right)>\operatorname{OFI}(T)$ since nonzero $\boldsymbol{P}_{T}(b)$ does not contribute to $\mathrm{OFI}(T)$, and $\boldsymbol{P}_{T^{\prime}}(a)$, which contributes to $\mathrm{OFI}\left(T^{\prime}\right)$, is larger than $\boldsymbol{P}_{T}(a)$. We can continue to construct a sequence with larger OFI in the same manner until the sequence has only element $a$ ( $L$ of them). Hence, $2-2 / N$ is an upper bound on OFI. FIVD value of such a sequence can then be computed as

$$
\frac{1}{(L-1)} \sum_{i=2}^{L} \frac{2-2 / N-\psi(i, N)}{\Psi(N)-\psi(i, N)}=\frac{1}{(L-1)} \sum_{i=2}^{L} 1=1 .
$$

\section{Discussions}

Since FIVD reflects short-term fairness, it implies the reflection of long-term fairness. According to the definition of FIVD given in (3), it is easy to see that FIVD can be applied to any population size except one, whose fairness issue is trivial. Hence, FIVD is independent of population size. By the definition of FIVD and Theorem 1, FIVD is good for any length of sequence (except $L=1$ ) and follows the intuition that the host with the lowest occurrence probability should
TABLE I

COMPARISON OF MAX/Min FAIRNESS INDEX, JAIN's INDEX, AND FIVD

\begin{tabular}{|c|c|c|c|c|}
\hline Sequence $(\mathrm{T})$ & $\mathrm{M} / \mathrm{m}$ & $J_{N}$ & $J_{2 N}$ & FIVD \\
\hline 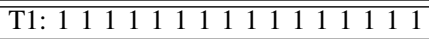 & $\infty$ & 0.25 & 0.25 & 1.00 \\
\hline T2: 11111111111111111111112 & $\infty$ & 0.26 & 0.25 & 0.99 \\
\hline 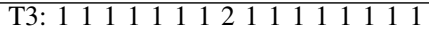 & $\infty$ & 0.29 & 0.31 & 0.92 \\
\hline  & 13 & 0.45 & 0.51 & 0.80 \\
\hline T5: 1111111234412341234 & 2.3 & 0.81 & 0.86 & 0.56 \\
\hline  & 2.3 & 0.82 & 0.85 & 0.27 \\
\hline (T7: 11111222222333334444 & 1 & 0.37 & 0.58 & 0.47 \\
\hline T8: 123312312231234444 & 1 & 0.63 & 0.79 & 0.17 \\
\hline T9: 1234123412341234 & 1 & 1.00 & 1.00 & 0 \\
\hline
\end{tabular}
in Several Sets of Sequences

be served in order to improve fairness. Hence, FIVD has the properties of symbol-level granularity. Furthermore, Theorems 2 and 3 show that FIVD assigns the minimum value 0 to any ideally fair sequence in $A_{T}(L)$. We term this property perfect representation. Finally, since FIVD uses occurrence probabilities and is tightly bounded between 0 and 1 , it is independent of the unit of measurement on the resource.

\section{Performance Evaluation}

In this section, we compare the max/min fairness index, Jain's index, and FIVD. We present our evaluations in numerical computations and ns2 simulations.

\section{A. Numerical Computations}

In our first numerical results, we compare FIVD with $\max / \mathrm{min}$ fairness and Jain's indices in Table I. The max/min fairness index was computed as the maximum occurrence among all elements divided by the minimum occurrence among all elements. Jain's indices, $J_{N}$ and $J_{2 N}$, were based on the sliding window Jain's index [6] with window size of $N$ and $2 N$, respectively. In these sequences, there are four hosts sharing a service $(N=4)$ and the sequence length is 16 ( $L=16)$. While the $\max / \mathrm{min}$ fairness index shows infinity for sequences $T 1, T 2$, and $T 3$, FIVD correctly shows that $T 3$ is the most fair among these three. This is because host 1 defers to host 2 in the middle of the sequence T3. FIVD confirms that $T 4$ is the most fair among the first four sequences.

The comparison between $T 5$ and $T 6$ further illustrates the effectiveness of FIVD to distinguish fairness among sequences. In $T 6$, the access of host 1 to the service is separated by those of the other hosts. Thus, $T 6$ is more fair than $T 5$, even though both have the same occurrence frequency as reported by $\mathrm{max} / \mathrm{min}$ fairness and Jain's indices. Interestingly, the sliding window Jain's index demonstrates some contradicting conclusions with different window sizes.

Sequences $T 7, T 8$, and $T 9$ provide insights on different ways to arrange the same set of elements. FIVD shows $T 9$ as the most fair sequence among the three, as one would expect. In contrast, the max/min fairness index treats them the same.

In Fig. 1, we present the changes of the OFI lower bounds $\psi(L, N)$ as a function of sequence length $L$ and number of hosts $N . \psi(L, N)$ demonstrates cycles of drops to zero coinciding with the cases when $L$ is a multiple of $N$. Furthermore, 


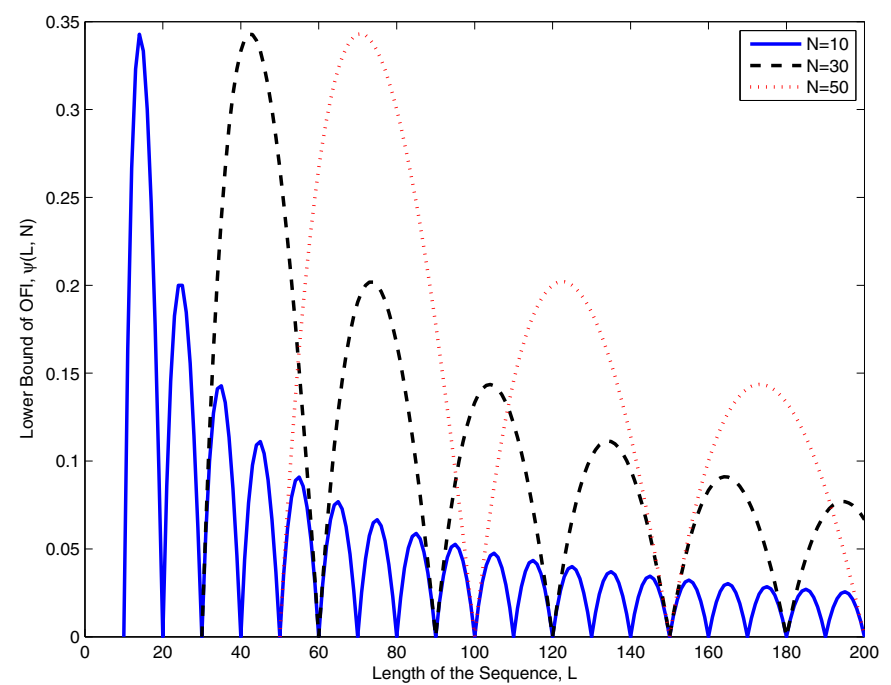

Fig. 1. Lower bound of OFI for a sequence with length of $L$ and $N$ hosts, $\psi(L, N) . \psi(L, N)=0$ when $L$ is a multiple of $N$. The curves all approach zero as $L$ increases.

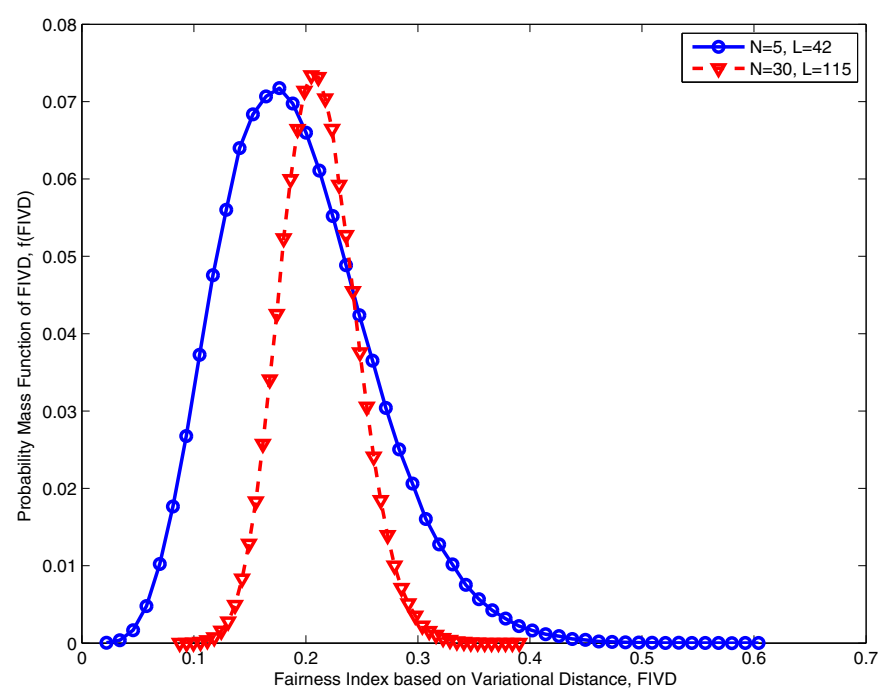

Fig. 2. The probability mass function of FIVD for randomly generated sequences of length $L$ and $N$ hosts.

the peak values of $\psi(L, N)$ for a fixed $N$ decreases as $L$ increases. This is because the effect of the last $\ell$ elements in the sequences diminishes as $L$ increases. Note the similarity among the curves for different $N$, suggesting that the curves are similar for the same ratio $L / N$.

We present the probability mass function of FIVD for randomly generated sequences of length $L$ and $N$ hosts in Fig. 2. These sequences were generated where each position chooses its element based on an i.i.d. uniform distribution among $N$ elements. We chose two pairs of parameters $(N, L)$ as $(5,42)$, and $(30,115)$. We intentionally chose $L$ values that are not multiples of $N$. Then we randomly generated 500,000 sequences and computed their FIVD values. The probability mass function has a similar shape to the Gaussian distribution,

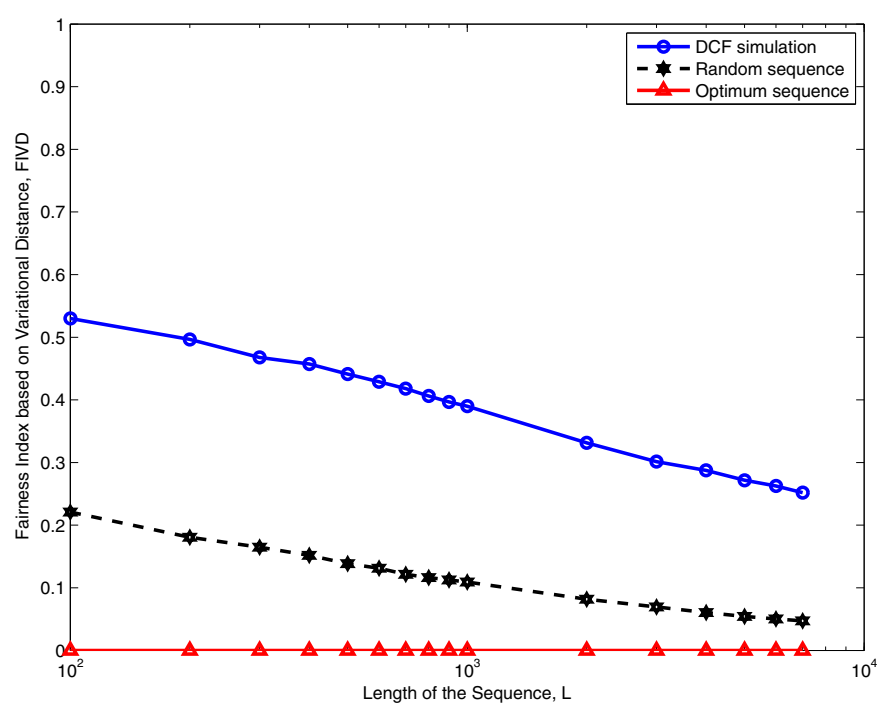

Fig. 3. FIVD of IEEE 802.11 DCF MAC scheme. We compare the FIVD values of a fully connected network with $N=30$ active senders for 100 seconds. The FIVD values are compared with that of random sequences of the same length and optimum sequences as suggested in Theorem 1 .

but they are bounded, as shown in Corollary 4.

Based on Fig. 2, we can see that it is rather unlikely to have a random sequence of length $L=115$ and $N=30$ with an FIVD value greater than 0.4 . However, most randomly generated sequences of length $L=42$ and $N=5$ have FIVD values between 0 and 0.5 .

\section{B. $n s 2$ Simulations}

In order to show the applicability of FIVD, we also ran simulations of the well-known IEEE 802.11 Distributed Coordinated Function (DCF) MAC in ns2 and investigated its fairness performance.

In Fig. 3, we plot the FIVD values of the IEEE 802.11 DCF MAC scheme with $N=30$ active senders for 100 seconds. The sequence of the successful senders was recorded and used to compute their FIVD values. For comparison purpose, we also randomly generated some sequences of the same length and calculated their FIVD values. These FIVD values are compared to that of the optimum sequence, which we have proven to have the minimum value of 0 .

It can be observed from Fig. 3 that the FIVD value of the transmission sequence is 0.3-0.5. Compared to the FIVD values of the random sequences of the same length, they are 2-5 times higher. For instance, the DCF transmission sequence of length $L=117$ and $N=30$ has an FIVD value around 0.5. According to our discussions in Fig. 2 of Section IVA, this value is outside the range of the FIVD values for randomly generated sequences. Therefore, we conclude that IEEE 802.11 DCF MAC does not provide fair services to the competing hosts.

We performed similar simulations and comparisons for a network with $N=5$ hosts and present the comparisons of FIVD values in Fig. 4. Interestingly, the FIVD values of IEEE 




Fig. 4. FIVD of IEEE 802.11 DCF MAC scheme. We compare the FIVD values of a fully connected network with $N=5$ active senders for 100 seconds. The FIVD values are compared with that of random sequences of the same length and optimum sequences as suggested in Theorem 1. Note that different position of the curve for IEEE 802.11 DCF MAC compared to $N=30$.

802.11 DCF MAC are smaller than those of random sequences. The DCF transmission sequence of length $L=42$ and $N=5$ has an FIVD value around 0.15 . According to our discussions in Fig. 2 of Section IV-A, this value lies within the FIVD values for randomly generated sequences. Therefore, we can conclude that the IEEE 802.11 DCF MAC scheme is fair to networks with small numbers of competing hosts, but not those with large numbers of competing hosts.

This result can be explained with the values of the minimum contention window (CWmin) in the IEEE 802.11 standard. CWmin is the initial contention window each competing host uses before it is doubled upon collisions. When the number of competing hosts is comparable to $\mathrm{CWmin}$, channel dominance is likely to take place. This, however, is unlikely to happen when there are only a few competing hosts, e.g., $N=5$.

\section{CONCLUSIONS}

We have presented a new fairness index, termed Fairness Index based on Variational Distance. FIVD computes the average of the occurrence fairness index that is based on the variational distance of the appearance frequency of different hosts and that of a uniform distribution, which is considered to give the most fair sequence. Through analysis and evaluations, we have shown that FIVD provides a much better fairness indication for competing hosts. Furthermore, since FIVD has the property of symbol-level granularity, it is possible to modify the resource allocation scheme on the fly based on the measured fairness. We leave this as a future work.

\section{ACKNOWLEDGMENT}

This work was supported in part by UNCG new faculty grant and by the National Science Council of Taiwan, R.O.C., under grants NSC 96-2221-E-305-002-MY3. Y. S. Han's work was partially support by his visit to LIVE lab at University of Texas at Austin.

\section{REFERENCES}

[1] A. K. Parekh and R. G. Gallager, "A generalized processor sharing approach to flow control in integrated services networks: the single-node case," IEEE/ACM Trans. Netw., vol. 1, no. 3, pp. 344-357, 1993.

[2] H. Luo, S. Lu, and V. Bharghavan, "A new model for packet scheduling in multihop wireless networks," in Proc. of the 6th annual ACM/IEEE international conference on Mobile computing and networking (MobiCom), August 2000.

[3] R. K. Jain, D.-M. W. Chiu, and W. R. Hawe, "A quantitative measure of fairness and discrimination for resource allocation and shared computer system," Technical Report DEC-TR-301, Digital Equipment Corporation, 1984.

[4] T. Ozugur, M. Naghshineh, P. Kermani, C. M. Olsen, B. Rezvani, and J. A. Copeland, "Balanced media access methods for wireless networks," in Proc. of the 4th annual ACM/IEEE international conference on Mobile computing and networking (MobiCom), 1998.

[5] S. Chan and M. Zukerman, "A new max-min fairness definition to neutralize malicious users," in Proc. of the 2000 IEEE International Conference on Communications (ICC), June 18-22 2000, vol. 3.

[6] C. E. Koksal, H. Kassab, and H. Balakrishnan, "An analysis of shortterm fairness in wireless media access protocols (poster session)," in Proceedings of the 2000 ACM SIGMETRICS international conference on Measurement and modeling of computer systems (SIGMETRICS), New York, NY, USA, 2000, pp. 118-119, ACM.

[7] G. Berger-Sabbatel, A. Duda, O. Gaudoin, M. Heusse, and F. Rousseau, "Fairness and its impact on delay in 802.11 networks," in Proc of the IEEE Global Telecommunications Conference (GLOBECOM), Nov. 2004, vol. 5, pp. 2967-2973.

[8] J. Eshet and B. Liang, "Randomly ranked mini slots for fair and efficient medium access control in ad hoc networks," IEEE Transactions on Mobile Computing, vol. 6, no. 5, pp. 481-493, 2007.

[9] C. Douligeris and L. N. Kumar, "Access to a network channel: a survey into the unfairness problem," in Proc. of SUPERCOMM/ICC '92. Discovering a New World of Communications, 1992, vol. 3.

[10] T. Nandagopal, T.-E. Kim, X. Gao, and V. Bharghavan, "Achieving MAC layer fairness in wireless packet networks," in Proc. of the 6th Annual ACM International Conference on Mobile Computing and Networking (MobiCom), August 6-11 2000.

[11] V. Kanodia, A. Sabharwal, B. Sadeghi, and E. Knightly, "Ordered packet scheduling in wireless ad hoc networks: mechanisms and performance analysis," in Proceedings of the 3rd ACM International Symposium on Mobile Ad Hoc Networking and Computing (MobiHoc), New York, NY, USA, 2002, pp. 58-70, ACM.

[12] J. Wong, J. Sauve, and J. Field, "A study of fairness in packet-switching networks," IEEE Trans. on Communications, vol. 30, no. 2, pp. 346-353, February 1982.

[13] F. P. Kelly, A. K. Maulloo, and D. K. H. Tan, "Rate control in communication networks: shadow prices, proportional fairness and stability," Journal of the Operational Research Society, vol. 49, 1998.

[14] M. Dianati, X. Shen, and S. Naik, "A new fairness index for radio resource allocation in wireless networks," in Proc. of the IEEE Wireless Communications and Networking Conference (WCNC), March 2005, vol. 2, pp. 712-717.

[15] T. M. Cover and J. A. Thomas, Elements of Information Theory, New York, NY: John Wiley and Sons, 1991. 